\title{
Methods Research and Software Development for Parameters Formalization of the Assignment Task Applicable to the Target Distribution
}

\author{
Denis Aleksandrovich Beloglazov, ${ }^{1}$ Valery Ivanovich Finaev ${ }^{(D},{ }^{1}$ \\ Victor Vladimirovich Soloviev, ${ }^{1}$ and Elena Nikolaevna Pavlenko \\ ${ }^{1}$ Southern Federal University, 105/42 Bolshaya Sadovaya Str., 344006 Rostov-on-Don, Russia \\ ${ }^{2}$ North Caucasus Federal University, 1, Pushkin Str., Stavropol, Russia \\ Correspondence should be addressed to Valery Ivanovich Finaev; vifinaev@sfedu.ru
}

Received 27 September 2019; Accepted 19 December 2019; Published 28 February 2020

Academic Editor: L. Fortuna

Copyright (C) 2020 Denis Aleksandrovich Beloglazov et al. This is an open access article distributed under the Creative Commons Attribution License, which permits unrestricted use, distribution, and reproduction in any medium, provided the original work is properly cited.

\begin{abstract}
The paper discusses the solution of the assignment task between two groups of mobile (MR) objects. The assignment task is to determine the purpose of MR to each other when playing football. This article analyzes well-known works devoted to the tasks of assignments, and the relevance of research in this area is noted. The task of target distribution is successfully solved with a small degree of uncertainty. The urgency of further research to solve the problem of target distribution in conditions of uncertainty is substantiated. The model of target assignment is considered. The basis of the model is a bipartite graph. The vertices of a bipartite graph are defined by the properties of MR. One group of MR is assigned red color and the other one group is blue. The properties of the mobile objects are formally determined by the membership grades given by the experts. Identification of a bipartite graph is a solution to the assignment problem. When the problem of target distribution on a bipartite graph is solved, the criterion of the maximum degree of bipartite is applied. Formulas are given to determine the degree of bipartite, taking into account the number of vertices in the graph and the weight of each edge in two fractions of the graph. The study of the target distribution model was carried out by the simulation method. A program is developed to solve the problem of assignments. Properties and degrees of belonging of properties of red and blue MR are set for carrying out researches. The program allows you to produce research and under equality of red and blue MR, and under their inequality. The analysis of modeling results is carried out.
\end{abstract}

\section{Introduction}

During the interaction of groups of mobile objects (MO), there is a task of assigning the mobile objects of one MO group to another group. For example, when simulating a fairly well-known task of robots playing football [1], robots of one group must interact with robots of another group. Another example of the application of the assigned task is the task of target assignment (the task of target distribution) between the individual groups [2] that is the robotic complex (RC). This task can be solved at different points of time at the beginning of the group motion and in the process of motion, in the latter case, even several times if it is necessary to evaluate the result of an individual mobile object activity and change the parameters of the task.
The task of target assignment in conditions of motion uncertainty, parameters and coordinates of targets and parameters of obstacles is relevant. If there is an absence of uncertainty, assignment tasks are successfully solved by the dynamic programming methods [3], then in conditions of uncertainty, these methods are difficult to apply. There are works $[4,5]$ in which assignment problems are solved by using expert knowledge, methods of the theory of fuzzy sets and fuzzy logic.

We will consider the interaction of MR groups provided that the problems of robot navigation in dynamic environments are solved, as well as the orientation control of MR. The solution of these problems are reviewed in many works, for example, in works [6-9]. A method for robot navigation in dynamic environments, referred to as visibility 
binary tree algorithm, is introduced for solving the MR navigation problem [6]. The strategy of control in hypercomplex algebra [7] can be applied for controlling the MR orientation in three-dimensional space. The assignment problem can be solved using methods of game theory. For example, in work [10], it is shown that two separate losing games MR can be combined following a random or periodic strategy in order to have a resulting winning game. However, this is a somewhat different aspect of the solution to the assignment problem, which should be considered in order to further develop the results of this paper.

It should also be said that the task of target assignment might not have an unambiguous solution. Thus, the task of target assignment is relevant.

\section{Innovative Strategies}

2.1. Analysis of Well-Known Works with the Assignment Task. In [11], the division of tasks and the assignment of the possibilities of using self-organization when presenting robotic groups as a swarm are proposed.

All these tasks are solved as smaller, solvable problems. These tasks can be solved in a self-organized way. Algorithms for self-organized assignment of tasks are proposed, and algorithms are tested in practical real-world applications. However, the authors themselves believe that the results are not generalized.

Groß et al. [12] give the solution to the problem of assignment between objects, taking into account market costs and benefits. Their study shows that in the conditions of a small degree of uncertainty, the problem is successfully solved. And with limited or inaccurate communications, it is rather difficult to obtain the adequate solutions.

Koeman et al. [13] consider the ways of using the communications between robotic systems for solving the object assignment task. A complex of robotic tools in which there is a collaboration between agents is realized. Several agents can work on this task. Algorithms for obtaining information are developed. Software is developed, and research is performed. It is shown that when solving the problems of object assignment, one can effectively use the power of an agent to achieve the targets.

The article [14] provides an overview of the well-known works in this area of work. It is made the analysis of the problems of cooperative robots. There are studies of the systems functioning from several autonomous mobile robots in order to study group architecture, resource conflicts, cooperation, training, and other tasks. The relevance and necessity of further solving the problems of cooperative robots, which is still in its infancy, is determined.

The article [15] considers the assignment between separate robot subsystems or individual group of robots of tasks of processing sensory information to control the joint operation of several manipulators with the coordination of their motion simultaneously in space and in time. The solution of the problem is considered in an uncertain environment for the optimal achievement of a group target.

The task of target assignment between robots is considered in $[1,2]$. Each mobile object in the group has its own task, and all these tasks constitute a single task. In [1, 2], a relative estimate of the efficiency of the $j$-th object of the $i$-th target problem is proposed. Relative estimates of the effectiveness of the solution are the additive components of the overall objective function. The task is reduced to the implementation of two successive operations. The first operation is object assignment by targets based on current information about the status of the robot and the distance to the target. The second operation is the assignment of the robot on the objectives in the next iterative step, taking into account the information on the objectives. A solution algorithm is proposed, but under uncertainty conditions, it is difficult to prove the convergence of this algorithm and evaluate its effectiveness.

In order to solve the problems of target assignment, fuzzy linear programming can be applied based on the Bellman-Zade decision model [16, 17]. The objective function and restrictions are in the form of fuzzy sets. Experts defined the membership function of the targets for fuzzy constraints and the membership function of the constraints. The condition is accepted that the function association of all fuzzy sets has a linear form. Then a maximizing solution is sought that ambiguously defines a clear solution. Decision-making algorithms have been developed for finding a clear optimal solution that maximizes the membership function of a fuzzy solution.

Accordingly, to the assignment task, it is paid a lot of attention to use robotic complexes. The solution of this task is successfully performed when there is sufficiently complete information about the robotic parameters in the group and about the objectives, as well as about the parameters of the external environment. However, in the conditions of incomplete source data, the solution of this problem is not easy and requires the application of expert knowledge and the corresponding software product.

2.2. Target Assignment Model. Graph theory uses methods for solving optimization problems. On the graph, the assignment task is reduced to a selected group of bipartite parts from a link graph. There are known solutions of the assignment problems using the methods of graph theory in fuzzy conditions defined by the fuzzy bipartite graphs. The task is solved by "selecting the optimal (by the defined criterion) maximum fuzzy part from the initial defined fuzzy graph" $[17,18]$.

It is possible to use the methods of graph theory for solving assignment problems with the fuzzy setting of parameters. This solution is limited to the condition of parameters formalization using bipartite graphs. Let us consider the features of the solution of the assignment problem with the use of fuzzy bipartite graphs when optimizing by the criterion of the maximum degree of bipartite. A fuzzy graph defined as the set of vertices is defined as a graph of $\widetilde{F}=\left\{\left\langle\mu_{F}<x_{i}, x_{j}\right\rangle /<x_{i}, x_{j} \gg\right\}, i, j=\overline{1, n}$. The degree of bipartite for any fuzzy dicotyledon part of the graph $\widetilde{G}^{\prime}=\left(V \cup V^{\prime}, \widetilde{F}^{\prime}\right), V \cup V^{\prime}=X, \widetilde{G}^{\prime} \subseteq \widetilde{F}$ is determined by

$$
d_{1}=1-\max \left(\mu_{\alpha}, \mu_{\beta}\right)
$$


where

$$
\begin{array}{ll}
\mu_{\alpha}=\max _{u \in U_{\alpha}} \mu_{u}, U_{\alpha}\left\{<x_{i}, x_{k}>\right\}, & x_{i}, x_{k} \in V \\
\mu_{\beta}=\max _{u \in U_{\beta}} \mu_{u}, U_{\beta}\left\{<x_{i}, x_{k}>\right\}, & x_{i}, x_{k} \in V^{\prime} .
\end{array}
$$

The degree of bipartite is determined by the formula, which takes into account the number of vertices in the graph and the weight of each edge in two fractions of the graph:

$$
d_{2}=1-\frac{\sum_{i=1}^{l} \mu_{e i}+\sum_{k=1}^{m} \mu_{e k}}{m_{V}-m_{V^{\prime}}},
$$

where $\mu_{e i}$ is the value of the membership function for the edge $e_{i} \in V ; \mu_{e^{\prime} k}$ is the value of the membership function for the edge $e_{k} \in V^{\prime} ; m_{V}$ is the number of edges between vertices $V$; and $m_{V^{\prime}}$ is the number of edges between vertices $V^{\prime}$ and

$$
\begin{gathered}
m_{V}=\frac{n_{V}\left(n_{V}-1\right)}{2}, \\
m_{V^{\prime}}=\frac{n_{V^{\prime}}\left(n_{V^{\prime}}-1\right)}{2},
\end{gathered}
$$

where $n_{V}$ is the number of vertices in the set $V$ and $n_{V^{\prime}}$ is the number of vertices in the set $V^{\prime}$.

If all possible dicotyledonous parts of a fuzzy graph that determine whether the robotic is in accordance with the objectives are considered, then it can find the degree of bipartite of these parts. After that, according to the results of the assessment, the solution of the assigned task is found. Estimation of the degree of bipartite of parts of a fuzzy graph can be made by the lower $d_{l}$ and upper $d_{u}$ estimates.

We can apply the algorithms for finding the maximum bipartite part of a fuzzy graph, allowing to select such a maximum bipartite part in the graph, the degree of bipartite which is greatest. As a result, the highest degree of compliance is established according to the selected criterion of the specific mobile object of the assigned target. There are several options for constructing these algorithms.

The algorithm $[19,20]$ can be applied for a clear graph based on a complete enumeration of all vertices and edges of the graph and extend its application to a fuzzy graph with the selection of the maximum bipartite parts of the graph.

It is possible to construct and apply an algorithm for extracting the maximum bipartite part of a fuzzy graph, in which the edges of a fuzzy graph are ranked in descending order of degrees of membership. Then an edge with the maximum degree of affiliation is taken, and one of its vertices is placed in the first lobe and the other vertex is placed in the other lobe. Then, in the order of ranking, the remaining edges are viewed, and their vertices are distributed by fractions of a fuzzy graph. After reviewing all the edges, the degree of bipartiteness of the resulting dichromatic fuzzy graph is calculated.

The computational complexity of the first algorithm for extracting the maximum bipartite part of a fuzzy graph is associated with the need to construct such several bipartite parts, which is determined by the number of vertices in the original part. The computational complexity of the second algorithm is associated with the number of noncontiguous edges in an ordered sequence. It is better to use the second algorithm if the ranking of the edges of the original fuzzy graph shows the absence of adjacent nonadjacent edges in the sequence.

It is considered that there are the mobile objects of one group, which are called the robotic complexes (RC) of red, and there are the mobile objects of another group, which are called RC blue. Let us set the task of how to assign the MO blue for the MO red in order to achieve success (win) by the MO red.

Fuzzy graph is set as $\widetilde{G}=(X, \widetilde{F})$. By the set $X=X_{1} \times X_{2}$, $X_{1}, X_{2} \in X, X_{1} \cup X_{2}=X, X_{1} \cap X_{2}=\varnothing$, where $X_{1}=\left\{x_{1}, x_{2}, \ldots\right.$, $\left.x_{m}\right\},\left|X_{1}\right|=m$ is the set of the red group, $X_{2}=\left\{x_{m+1}, x_{m+2}\right.$, $\left.\ldots, x_{n}\right\},\left|X_{2}\right|=m$ is the set of the blue group. The fuzzy graph $\widetilde{F}$ is determined from the properties of the elements of the set $X$, which are taken from the MO parameters of the groups of red and blue, specified both in a clear and in a fuzzy form. All MO players are identified, that is why their parameters are known.

The properties of the robotic elements of the players of the red group are determined by the set $P_{1}=P_{1}^{1}, P_{1}^{2}, \ldots, P_{1}^{m}$, where 1 is the index of the membership of the set of the red MO properties, for each $i$-th $\mathrm{MO}$ of the red the set of properties is determined $P_{1}^{i}=\left\{p_{11}^{i}, p_{12}^{i}, \ldots, p_{1 r_{i}}^{i}\right\}, \quad i=\overline{1, m}$.

Similarly, let us determine the set $P_{2}$ of the blue MO $P_{2}=P_{2}^{1}, P_{2}^{2}, \ldots, P_{2}^{n}$, where 2 is the index of the membership of the set of properties of blue football team, for each $j$-th blue MO, the set of properties is defined $P_{2}^{j}=\left\{p_{21}^{j}, p_{22}^{j}, \ldots, p_{2 r_{j}}^{j}\right\}, \quad i=\overline{1, n}$.

Let every robot be a robot soccer player. To set the properties of the red and blue RC, the following characteristics are applicable: 1 -the indicator of gains from an opponent football player; 2-the accuracy of hitting the sword; 3-the motion speed; 4-the effectiveness of the vision system; 5 -the long-range characteristic of hitting the sword; 6-the positive effect of inertia; 7-the protection from the power of the opponent; 8-the protection from interference; and 9-the information security.

Thus, all the parameters of the target assignment problem are considered, in general, as fuzzy, defined by a pair of $\quad \tilde{p}_{k}^{i}=\left\{\left\langle\mu_{p_{k}^{i}}\left(d_{k}\right) /\left(d_{k}\right)\right\rangle\right\}, \quad d_{k} \in D_{k} k=\overline{1, g}$, where $D_{k}$ is the base set for determining a fuzzy parameter $\widetilde{p}_{k}^{i}$, and $\mu_{p_{k}^{i}}\left(d_{k}\right)$ is the membership function of the $d_{k}$ element of a fuzzy set $\tilde{p}_{k}^{i}$.

Let us give an example of setting the properties for mobile objects of a group of red and blue. Both red and blue groups have four mobile objects.

The results of the expert assignment of the properties membership grades for each mobile object of the group of red and blue are shown in Table 1.

As a result, we obtained the initial plan of the assignment task of target assignment in the form of a bipartite graph, which is shown in Figure 1.

In Figure 1, on the left, there are the vertices of the graph corresponding to the robotic numbers of the red group, and to the right of the graph, there are the vertices corresponding 
TABLE 1: Results of expert assignment of membership assessment properties for each mobile object of a red and blue group.

\begin{tabular}{|c|c|c|c|c|c|c|c|c|}
\hline \multirow{2}{*}{ No. of property } & \multicolumn{4}{|c|}{ Number of objects in the red team } & \multicolumn{4}{|c|}{ Number of objects in the blue team } \\
\hline & 1 & 2 & 3 & 4 & 1 & 2 & 3 & 4 \\
\hline 1 -win rate & 0,7 & 0,9 & 0,8 & 0,95 & 0,9 & 0,7 & 0,8 & 0,8 \\
\hline 2-hit accuracy & 0,95 & 0,7 & 0,8 & 0,7 & 0,7 & 0,8 & 0,8 & 0,7 \\
\hline 3-motion speed & 0,8 & 0,9 & 0,9 & 0,8 & 0,8 & 0,8 & 0,9 & 0,6 \\
\hline 4 -efficiency of the vision system & 0,8 & 0,9 & 0,8 & 0,8 & 0,8 & 0,7 & 0,7 & 0,8 \\
\hline 5-long-range characteristic of hitting the sword & 0,9 & 0,9 & 0,8 & 0,7 & 0,7 & 0,8 & 0,8 & 0,7 \\
\hline 6 -inertia & 0,6 & 0,7 & 0,6 & 0,8 & 0,6 & 0,7 & 0,7 & 0,6 \\
\hline 7 - protection from the power of the opponent & 0,6 & 0,8 & 0,7 & 0,9 & 0,5 & 0,7 & 0,7 & 0,7 \\
\hline 8 - protection from interference & 0,7 & 0,5 & 0,6 & 0,6 & 0,5 & 0,7 & 0,8 & 0,5 \\
\hline 9-information security & 0,9 & 0,9 & 0,8 & 0,7 & 0,7 & 0,6 & 0,9 & 0,9 \\
\hline
\end{tabular}

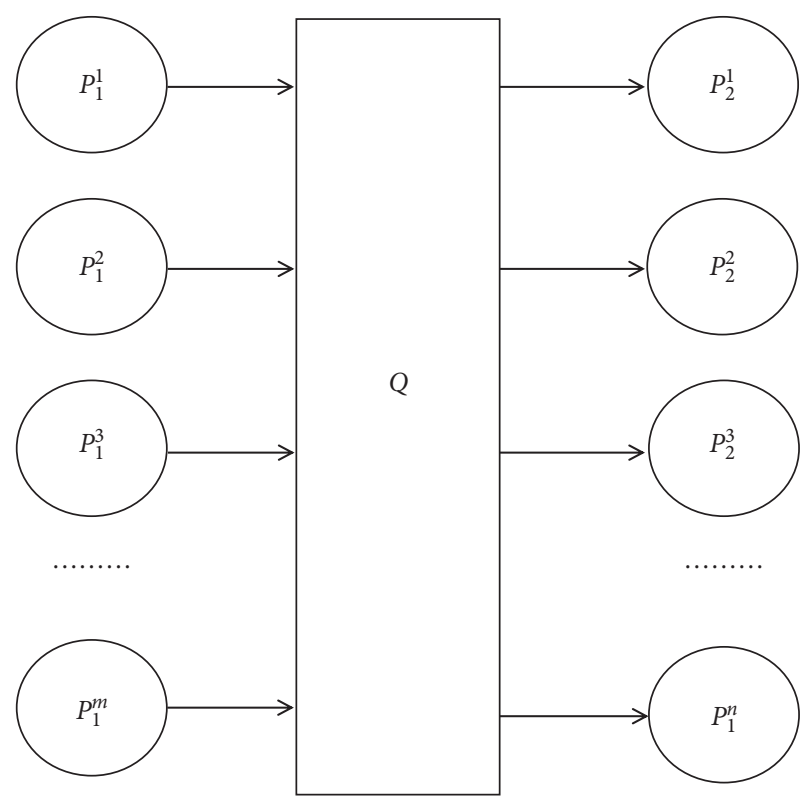

FIgURE 1: Initial plan for the assignment task.

to the robotic numbers of the blue group. The task of target assignment is associated with finding a match $q=\left\langle P_{1}, P_{2}, Q\right\rangle$, where $P_{1}$ is the departure area of the correspondence, $P_{2}$ is the arrival area, and $Q$ is the correspondence graph, generally a fuzzy graph that should be identified to solve the target assignment task. The correspondence graph is in the form of a set:

$$
\begin{aligned}
Q=\{ & <1_{\mathrm{r}}, 1_{\mathrm{b}}, \mu\left(1_{\mathrm{r}}, 1_{\mathrm{b}}\right)>,<1_{\mathrm{r}}, 2_{\mathrm{b}}, \mu\left(1_{\mathrm{r}}, 2_{\mathrm{b}}\right)>, \ldots, \\
& <1_{\mathrm{r}}, n_{\mathrm{b}}, \mu\left(1_{\mathrm{r}}, n_{\mathrm{b}}\right)>,<2_{\mathrm{r}}, 1_{\mathrm{b}}, \mu\left(2_{\mathrm{r}}, 1_{\mathrm{b}}\right)>, \\
& <2_{\mathrm{r}}, 2_{\mathrm{b}}, \mu\left(2_{\mathrm{r}}, 2_{\mathrm{b}}\right)>, \ldots,<2_{\mathrm{r}}, n_{\mathrm{b}}, \mu\left(2_{\mathrm{r}}, n_{\mathrm{b}}\right)>, \ldots, \\
& <m_{\mathrm{r}}, 1_{\mathrm{b}}, \mu\left(m_{\mathrm{r}}, 1_{\mathrm{b}}\right)>,<m_{\mathrm{r}}, 2_{\mathrm{b}}, \mu\left(m_{\mathrm{r}}, 2_{\mathrm{b}}\right)>, \\
& \left.<m_{\mathrm{r}} n_{\mathrm{b}}, \mu\left(m_{\mathrm{r}} n_{\mathrm{b}}\right)\right\},
\end{aligned}
$$

where $\mu\left(i_{\mathrm{r}}, j_{\mathrm{b}}\right)$ is the membership degree (correspondence) of the $j$-th blue MO to the $i$-th red MO in solving the task of target assignment.

The definition of values $\mu\left(i_{\mathrm{r}}, j_{\mathrm{b}}\right)$ can be performed by an expert with known tactical and technical data of the red and blue football players.
The elements of the $P_{1}$ set can be divided into disjoint subsets that characterize the maneuverability, the possibility of winning the opponent, the reliability, and so on. For example, the nine properties in Table 1 can be divided into two subsets $S_{1}=\langle 1,2,3,4,5\rangle$ and $S_{2}=\langle 6,7,8,9\rangle, S_{1} \cap S_{2}=S$ is the set of the MO properties of the group.

The subset $S_{1}$ contains "active" properties aimed at improving the efficiency of achieving a win, and the subset $S_{2}$ contains "passive" properties that provide protection against a loss but restraining the achievement of the winning. Such a division of properties reflects the subjective point of view. It is useful because, with many of the properties under consideration, one can combine them and look for a more simplified way of solving the task of target assignment.

The requirements can be also formulated in a form of lower values of parameters (properties) of a robotic group or each MR separately. Then, based on the chosen decisionmaking model [21], it is possible to draw a conclusion about the effectiveness of the created robotic group and the individual robot.

The basis for building a graph of correspondence $Q$ is the dominance relation (preference) between the elements $X_{1}$ of the robotic set of the red group and $X_{2}$ of the robotic set of the blue group. This domination relation must be identified when solving the task of assignment. Taking into account the membership grades, it is obtained the plan of the assignment task of target assignment in the form of a fuzzy bipartite graph, the general view of which is shown in Figure 2.

(i) Definition: two fuzzy relations $\alpha=\left\{u, \mu_{\alpha}(u)\right\}$ and $\beta=\left\{u, \mu_{\beta}(u)\right\}$ are equal, i.e., $\alpha=\beta$ if $\mu_{\alpha}(u)=\mu_{\beta}(u)$ for any $u \in U$ [22].

(ii) Fuzzy dominance: the fuzzy relation $\alpha$ is a fuzzy subset of the fuzzy relation $\beta$, i.e., $\alpha \subseteq \beta(\alpha \subset \beta)$ when $\mu_{\alpha}(u) \leq=\mu_{\beta}(u)\left(\mu_{\alpha}(u)<\mu_{\beta}(u)\right)$ for any $u \in U$. It is sometimes said that $\beta$ includes (dominates) $\alpha$.

Fuzzy inclusion is determined by applying the degree of fuzzy inclusion for each $j$-th fuzzy set $\widetilde{P}_{2}^{j}=\left\{p_{21}^{j}, p_{22}^{j}, \ldots\right.$, $\left.p_{2 r_{j}}^{j}\right\}, i=\overline{1, n}$ into every $i$-th fuzzy set $\widetilde{P}_{1}^{i}=\left\{p_{11}^{i}, p_{12}^{i}, \ldots\right.$, $\left.p_{1 r_{i}}^{i}\right\}, i=\overline{1, m}$ according to the formula provided that the power of these sets is equal $(m=n)$ :

$$
v\left(\widetilde{P}_{2}^{j}, \widetilde{P}_{1}^{j}\right) \cap_{j}\left(\mu\left(p_{2 i}^{j}\right) \longrightarrow \mu\left(p_{1 i}^{j}\right)\right), \quad i=\overline{1, m},
$$




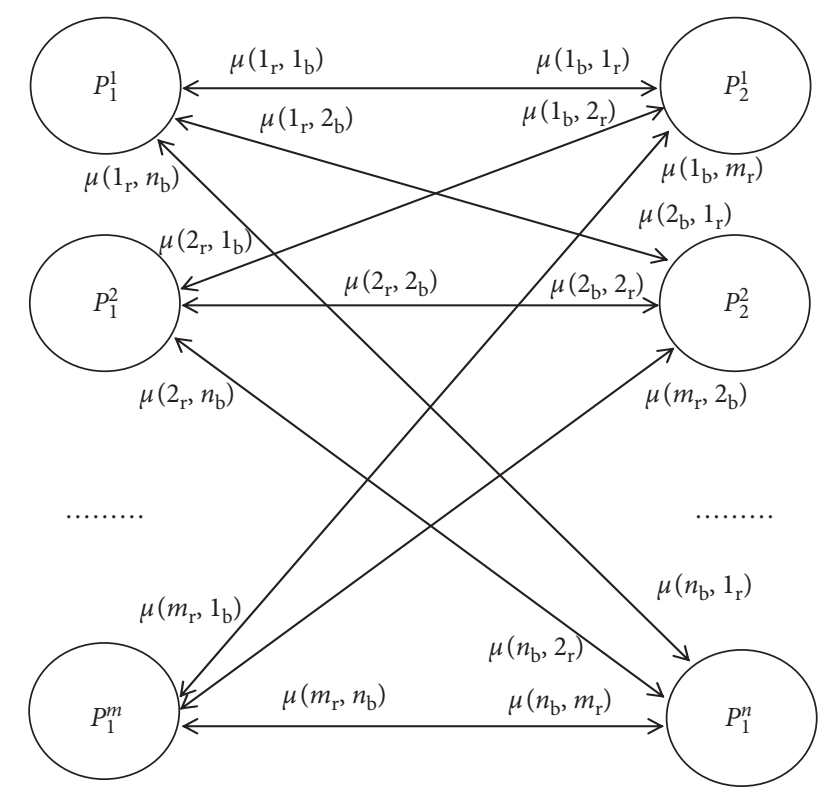

FIGURE 2: Interpretation of the task of target assignment in the form of a fuzzy bichromatic graph.

where the operation of fuzzy implication is performed in the Zada basis: $\widetilde{A} \longrightarrow \widetilde{B}=\max (1-\widetilde{A}, \widetilde{B})$.

Studies of the target assignment model can only be carried out using simulation methods, since there is a formal task of finding the optimal solutions, but in practice it is very difficult and even impossible to obtain analytical solutions.

\section{Results}

3.1. Software for Solving Assignment Tasks. The software does not require data exchange with external systems, which makes it not difficult to implement. The interaction between software modules occurs through common system data.

When working with the software, the input parameters in the form of numerical values of each object for the selected MO properties should be entered strictly less than 1 and separated by commas. The number of software in the group cannot be equal to zero. Output parameters (results of the obtained solutions) are presented in the form of a matrix of values and the directly resulting value.

As noted above, the simulation is performed for two MO groups, blue and red, playing football against each other. It should be assigned to the red $\mathrm{MO}$ corresponding to the blue $\mathrm{MO}$ in the game so that the red MO can win.

When you start the software, the main modal window appears, which is shown in Figure 3.

In the main modal window, the task is performed:

(i) The number of red robots

(ii) The number of blue robots

(iii) The selection of the compared properties of the red and blue MO groups is carried out

The software can do research not only in the situation of equality of elements, but also in any other situations. After entering the data, it must be clicked the "Apply" button to continue the work of the program or "Reset" if the information is entered incorrectly and then it must be corrected.

In the dialog box shown in Figure 4, it must be entered the numerical characteristics of the properties of each robot in accordance with the sequence number of the selected property. The numerical characteristic of the property is a membership grade, which is set by the expert. In the window on the left, there are the properties values of the red robotic group and on the right the blue one.

The next step is to select the robot sequence number of the group with which it will be necessary to make a comparison (see Figure 5).

As a result of the software operation, it is obtained the solution found in the form of a table shown in Figure 6 for the first $\mathrm{MO}$ and in Figure 7 for the second MO of the red group.

\section{Discussion}

4.1. Analysis of the Results of Solving the Task of Target Assignment. We will conduct three experiments and compare the results of solutions under a priori conditions:

(i) Red group is weaker than blue group of mobile objects

(ii) Red group is stronger than blue group of mobile objects

(iii) Mobile objects of red are approximately equal in strength to blue ones

4.1.1. Experiment ${ }^{\#} 1$. In each group, there are 4 red robots and 4 blue robots. Let us choose properties for comparison: the range characteristic of hitting the sword, inertia, protection from the forceful impact of a football opponent (see Figure 8).

To simplify the names of the properties in the results table are replaced by numbers in accordance with the sequence number among the selected properties, i.e., 1-longrange characteristic of hitting the sword, 2-inertia, 3 -protection from the forceful impact of the opponent football player (see Figure 9, the entered values of the degrees of property belonging).

In the first experiment, it is determined a priori that the red robotics are "weaker" than the blue robotics, and the solution of the target assignment problem is obtained, shown in Table 2.

In Table 2, it is shown that there is not a single degree of affiliation greater than 0.5 . Consequently, with such parameters of the red robotic objects, their opposition against the blue robotic objects will not give a win in the game.

4.1.2. Experiment ${ }^{\#}$ 2. Let the properties, listed in Figure 10, of the first red group of robotic systems to be noticeably superior to the properties of the second blue group of robotic systems. In Figure 10, there are shown the values of the entered property grade values. In Table 3 is shown the result of the obtained solution of the target assignment problem. 


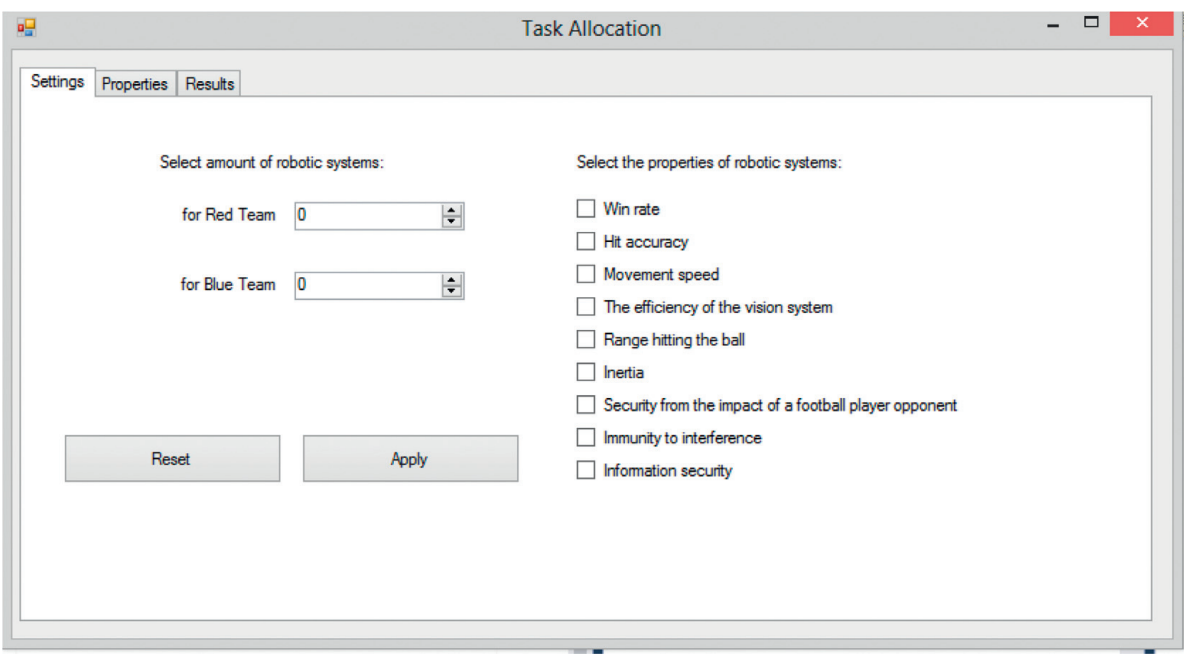

Figure 3: Main software modal window.

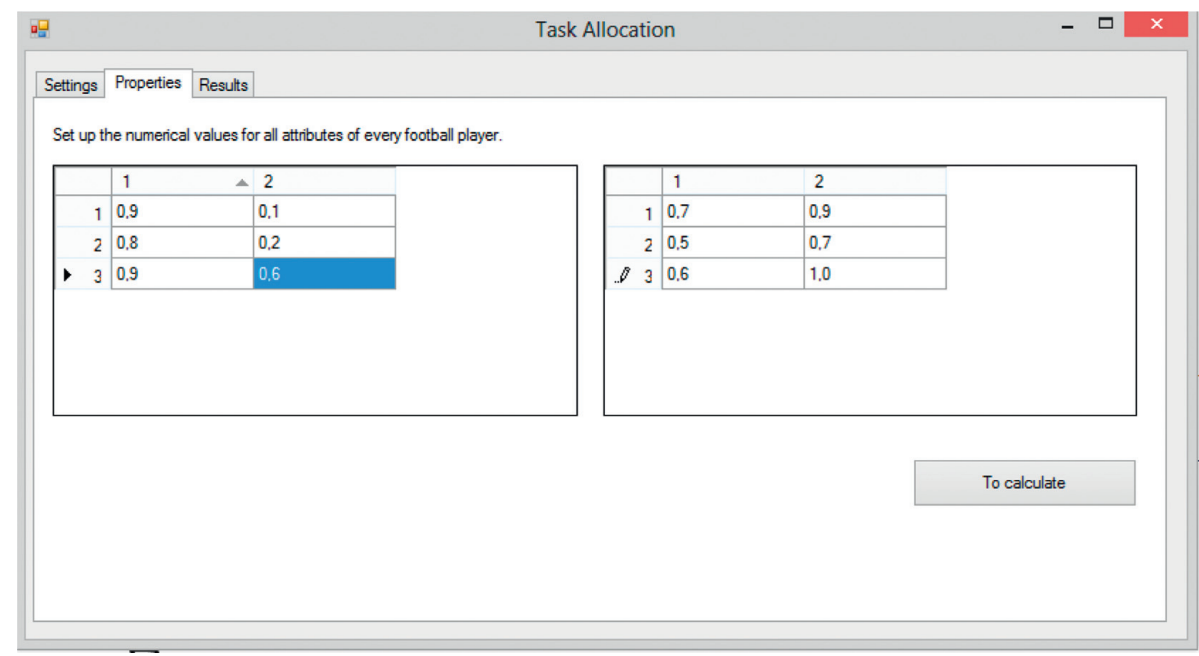

FiguRE 4: Entering the numerical characteristics of the properties for each robot.

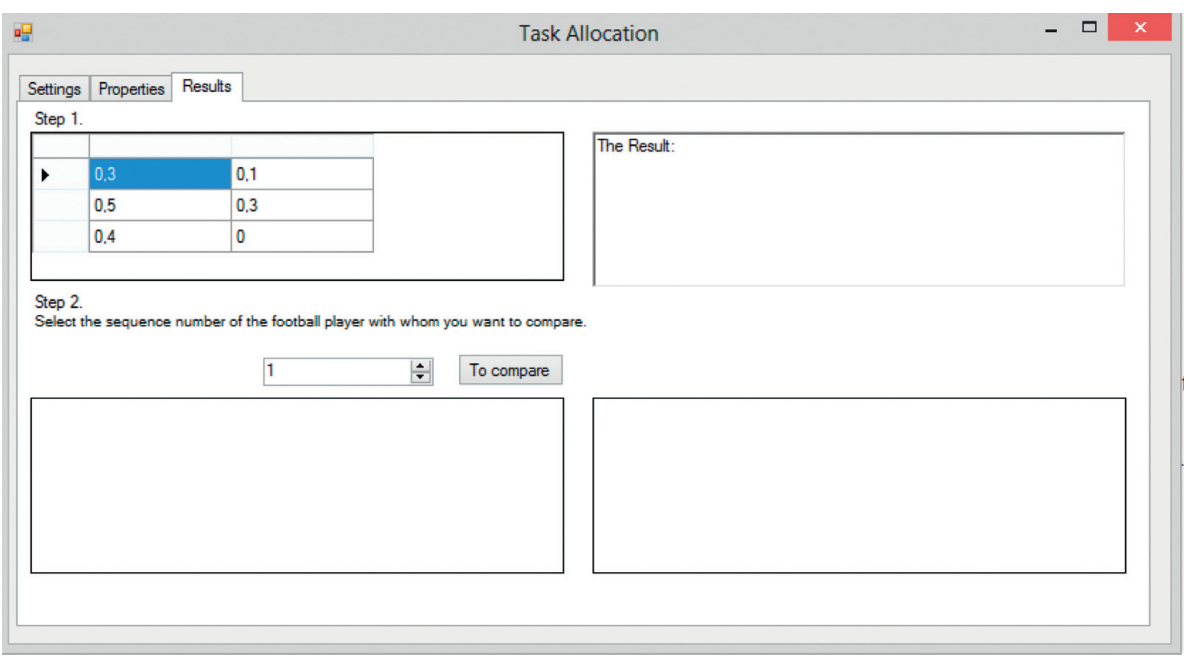

FIGURE 5: Selection of the sequence number of the MO group. 


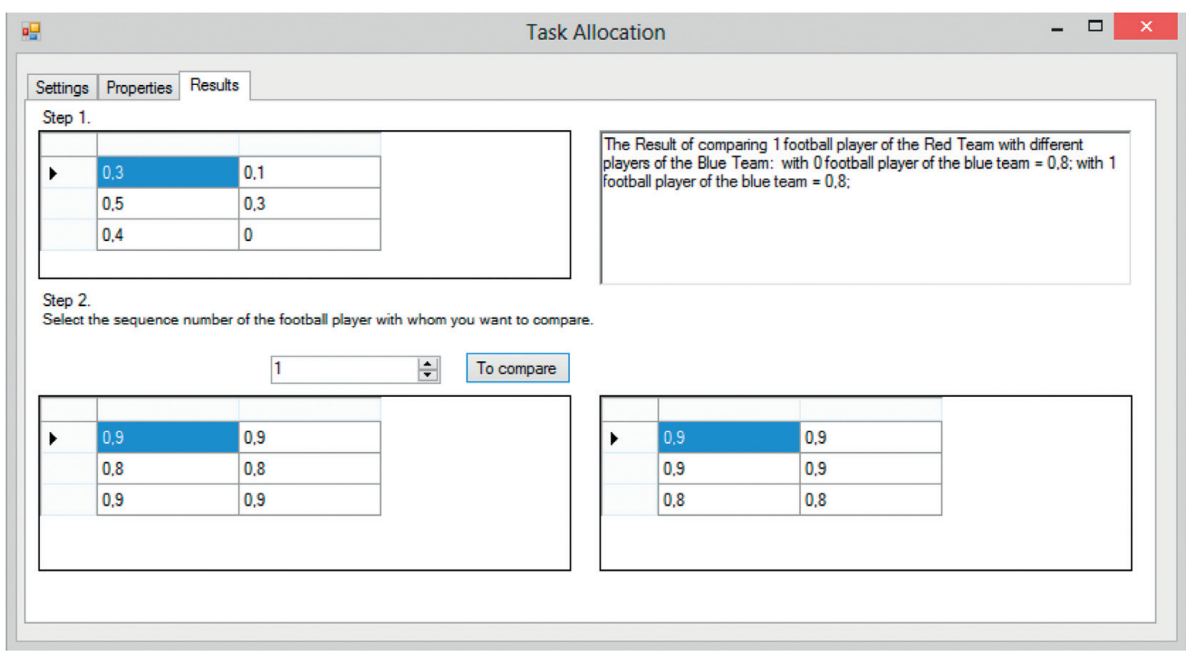

FigURE 6: Result of the solution for the first MO of the red group.

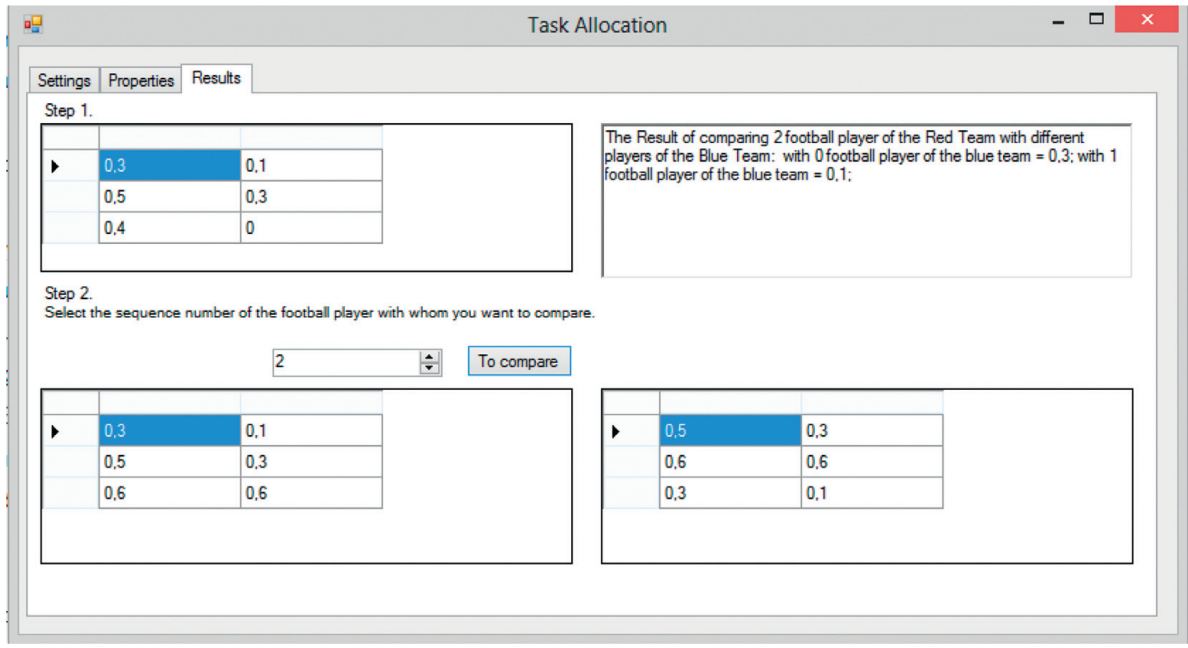

FigURE 7: Result of the solution for the second MO of the red group.

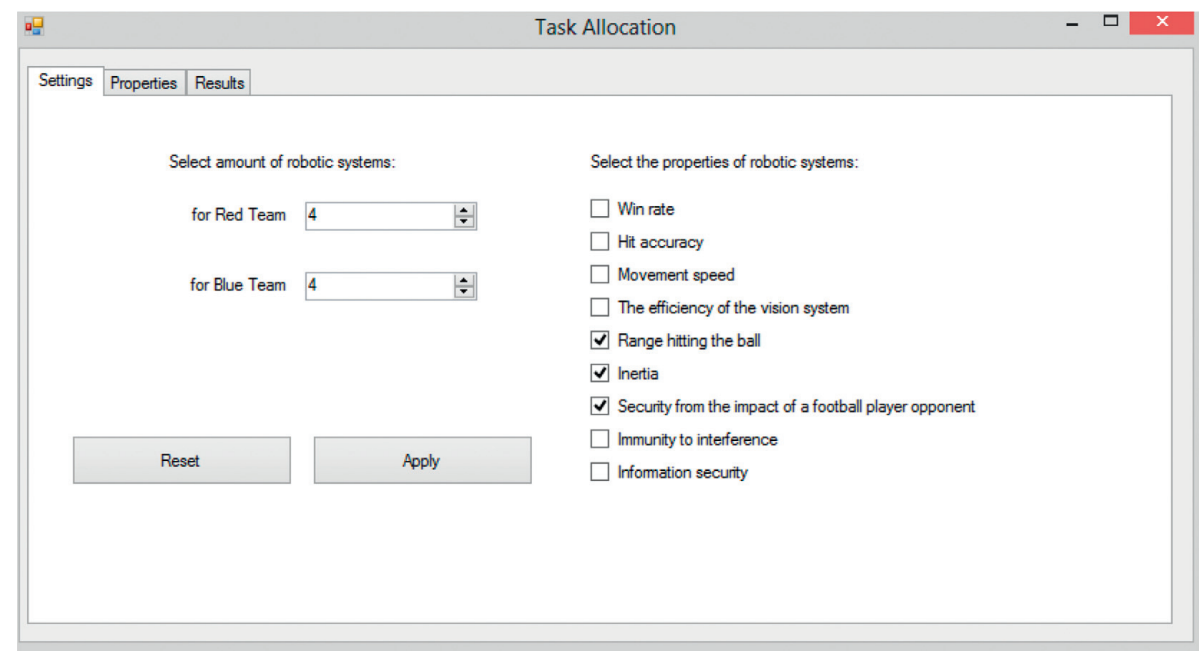

FIGURE 8: Selected robotics options. 


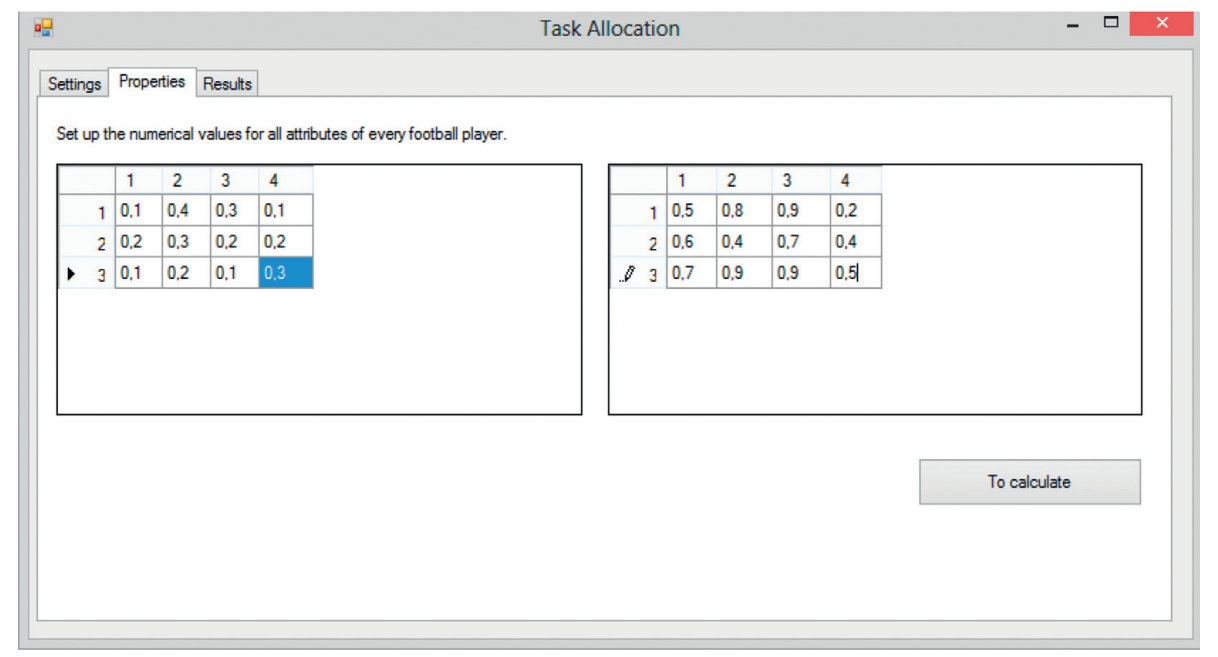

Figure 9: Entering the values of the robotic properties of the group of red and blue in the first experience.

TABLE 2: Solving the goal assignment problem in the first experiment.

\begin{tabular}{lcccr}
\hline & 1 st & 2nd & 3rd & \\
\hline 1st & 0,3 & 0,3 & 0,3 & 0,3 \\
2nd & 0,1 & 0,2 & 0,1 & 0,2 \\
3rd & 0,1 & 0,2 & 0,1 & 0,1 \\
4th & 0,1 & 0,2 & 0,1 & 0,3 \\
\hline
\end{tabular}

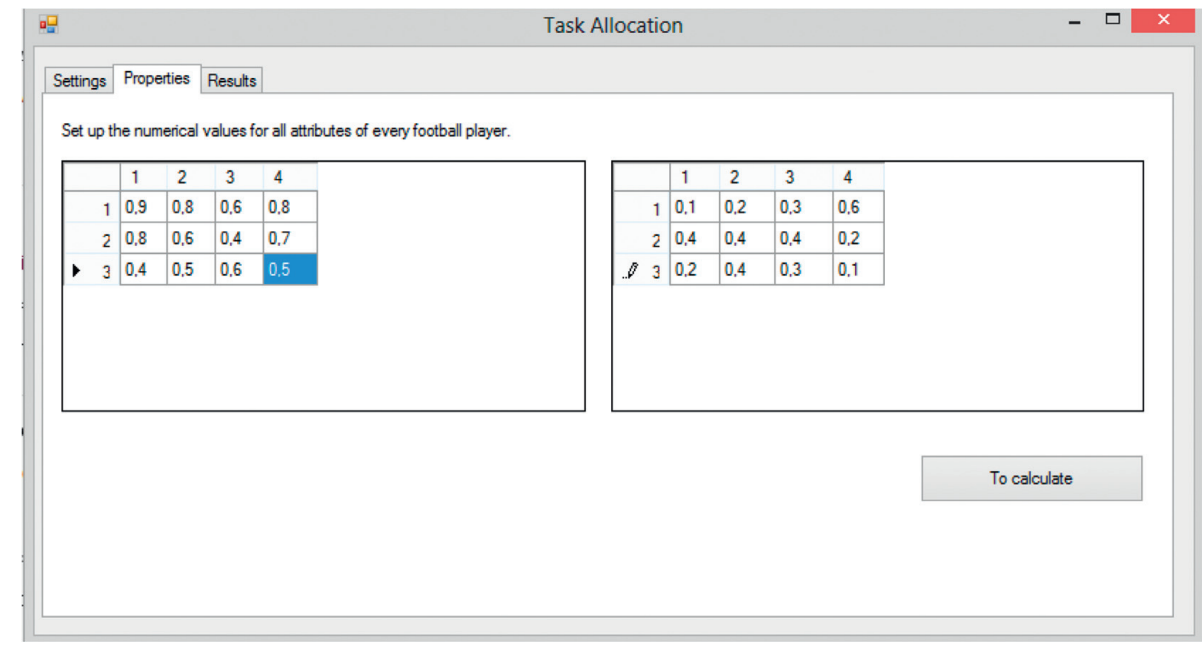

FIGURE 10: Entering the values of the robotic properties of the group of red and blue in the second experiment.

TABLE 3: The result of the solution to the goal assignment problem.

\begin{tabular}{lcccr}
\hline & 1 st & 2nd & 3rd & \\
\hline 1st & 0,8 & 0,6 & 0,6 & 0,7 \\
2nd & 0,6 & 0,6 & 0,6 & 0,6 \\
3rd & 0,7 & 0,6 & 0,6 & 0,7 \\
4th & 0,8 & 0,8 & 0,8 & 0,8 \\
\hline
\end{tabular}




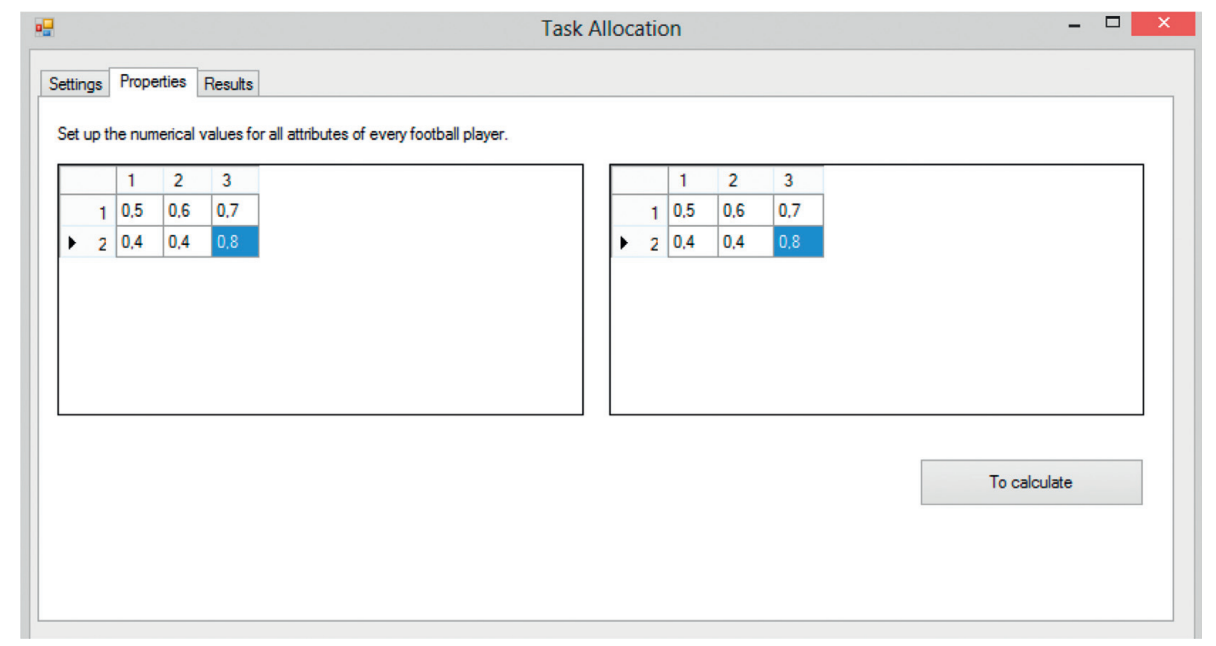

FIGURE 11: Entering the values of the mobile objects' properties of the group of red and blue in the third experiment.

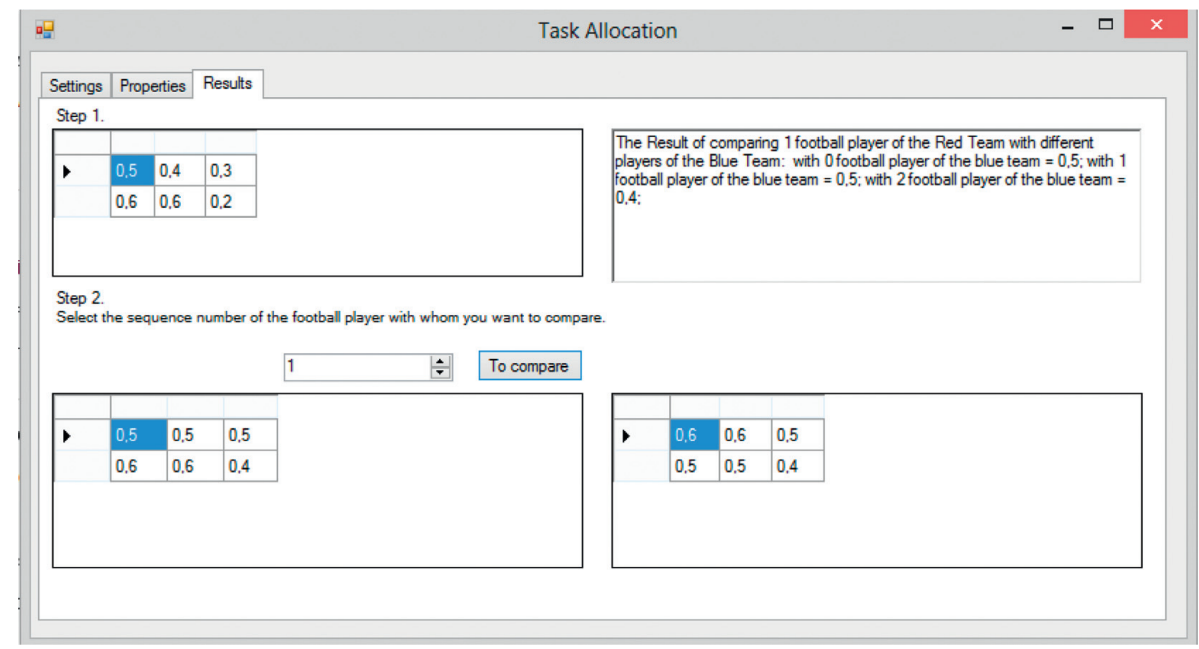

Figure 12: Obtained values of the comparison of the 1st mobile object of red with the blue ones and the identification of the most successful purpose.

The decision shows that the second blue robotic systems can be assigned to the first red robotic systems, since it is "significantly weaker than the first red robotic group" and the first red mobile objects will win against the second blue team.

4.1.3. Experiment \#3. Select the degree of belonging of properties in such a way that the red and blue teams have approximately the same potential. Let there be 3 robotics of groups of red and blue. Therange characteristic of hitting the sword and the speed of the mobile objects are considered, as shown in Figure 11. The results of the solution in the third experiment are shown in Figures 12, 13, and 14.

The final decision on the appointment is summarized in Table 4.

From Table 4, it follows that the third robot of the blue group should be assigned to the third robot of the red group and the first and second robots of the red group would be an equivalent appointment against the remaining blue robots.
4.2. Conclusion. The task of the target assignment refers to the study of operations for the class of assignment tasks. With sufficient knowledge of objects, this problem can be solved by linear (nonlinear) programming methods. Under uncertainty, such problems are proposed to be solved using the methods of graph theory and the theory of fuzzy sets. A model has been developed for solving the assignment problem. The difference of the model is in the task and in the identification of a fuzzy domination relation on a bipartite graph, which makes it possible to find appointments for the soccer players of the red MR and the blue MR. Robotic characteristics are defined by a set of properties that are formally defined by degrees of ownership. The degree of ownership is set by experts. Studies conducted with boundary values of the degrees of belonging of robotic properties showed adequate work of the developed program for solving the task of the target assignment.

Further research in this area is related to targeting in the presence of obstacles in the environment of movement. The coordinates of the obstacles may be unknown in advance. 


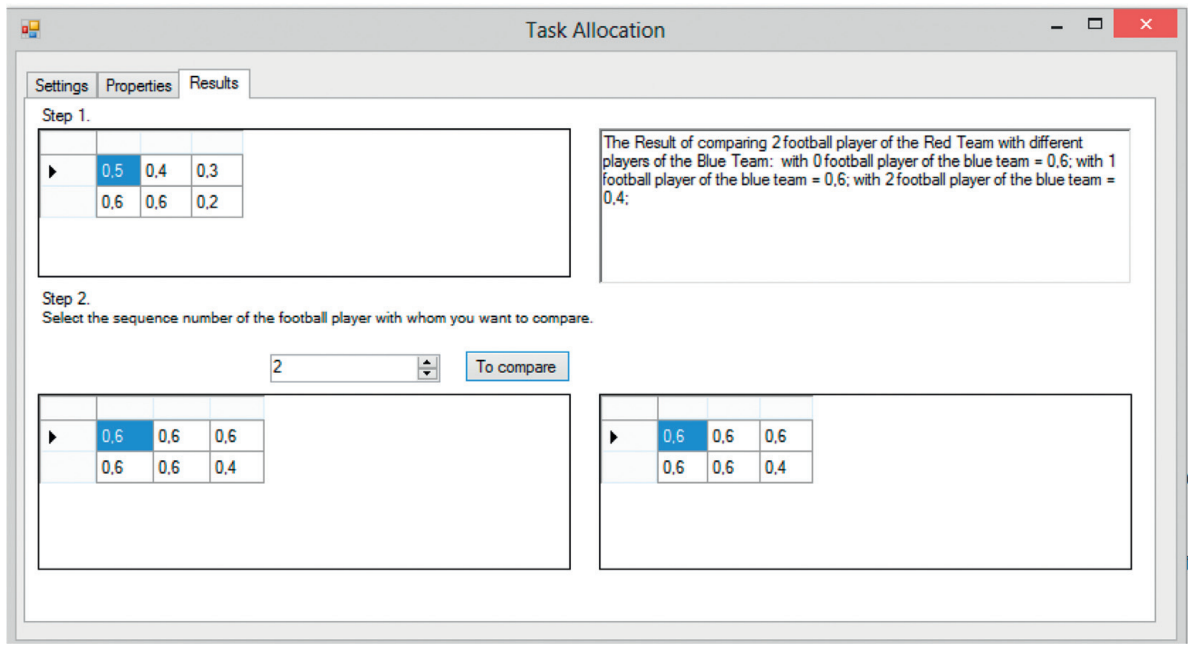

FIGURE 13: Obtained values of the comparison of the 2nd mobile object of the team of red with the team of blue and the identification of the most successful purpose.

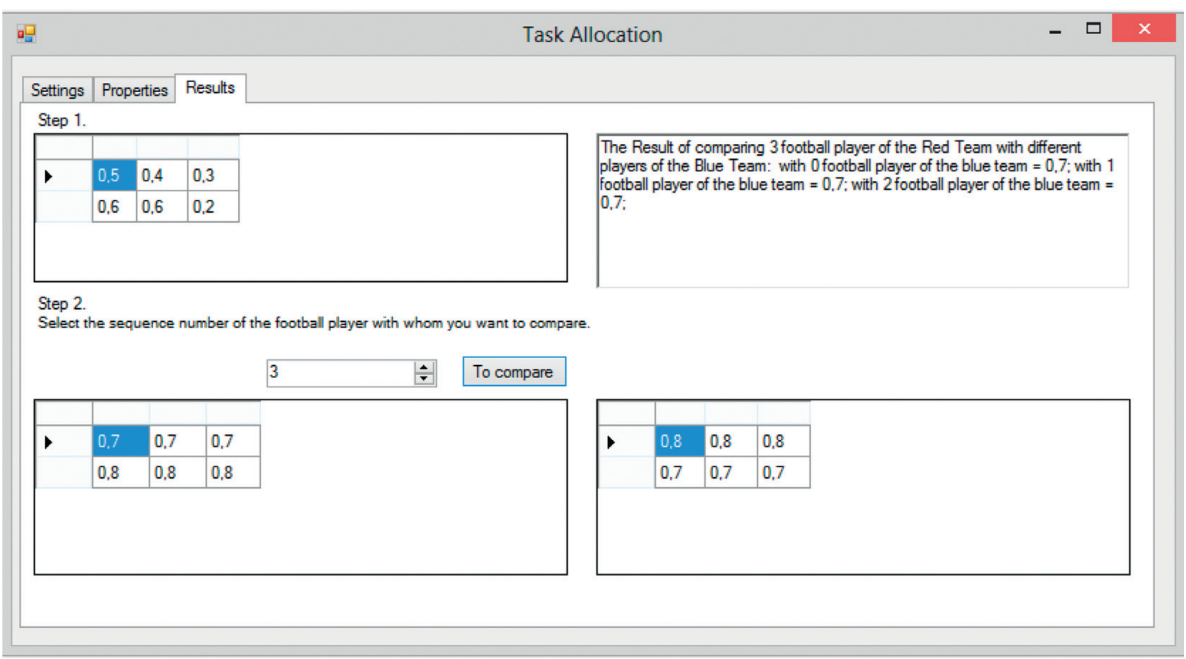

Figure 14: Obtained values of the comparison of the 3rd mobile object of the group of red with the robotic of blue and the identification of the most successful targeting.

TABLe 4: The final decision on the appointment.

\begin{tabular}{lccc}
\hline & 1 st & 2nd & 3rd \\
\hline 1st & 0,5 & 0,6 & 0,7 \\
2nd & 0,5 & 0,6 & 0,7 \\
3rd & 0,4 & 0,4 & 0,7 \\
\hline
\end{tabular}

\section{Data Availability}

No data were used to support this study.

\section{Conflicts of Interest}

The authors state no conflicts of interest.

\section{Authors' Contributions}

All authors contributed to experiments and writing of this paper.

\section{Acknowledgments}

The study was supported by the RSCF grant 18-19-00621, from the joint stock company "Scientific-Design bureau of Robotics and Control Systems."

\section{References}

[1] J. Zhong, T. Nakashima, and H. Akiyama, "A study on the analysis of soccer games using distributed representation of actions and players," ICIC Express Letters, vol. 13, no. 4, pp. 303-310, 2019.

[2] Y. Marchukov and L. Montano, "Fast and scalable multi-robot deployment planning under connectivity constraints/19th," in Proceedings of the IEEE International Conference on Autonomous Robot Systems and Competitions, ICARSC, Gondomar, Portugal, April 2019, https://www2.scopus.com/inward/ record.uri?eid $=2$-s2.0-85068437160\&doi $=10.1109 \%$ 2fICARSC.2019.8733637\&partnerID=40\&md5=DOI:10. 1109/ICARSC.2019.8733637. 
[3] A. Visentin, S. D. Prestwich, R. Rossi, and A. Tarim, "Modelling dynamic programming-based global constraints in constraint programming," Advances in Intelligent Systems and Computing, vol. 991, pp. 417-427, 2020.

[4] H. Gunawan and H. Ramadhan, "Increased accuracy of selection high performing employees using multi attribute utility theory (MAUT)," in Proceedings of the 2018 6th International Conference on Cyber and IT Service Management (CITSM), Parapet, Indonesia, August 2018.

[5] J. L. García-Lapresta and R. González Del Pozo, “An ordinal multi-criteria decision-making procedure under imprecise linguistic assessments," European Journal of Operational Research, vol. 279, no. 1, pp. 159-167, 2019.

[6] A. T. Rashid, A. A. Ali, M. Frasca, and L. Fortuna, "Path planning with obstacle avoidance based on visibility binary tree algorithm," Robotics and Autonomous Systems, vol. 61, no. 12, pp. 1440-1449, 2013.

[7] L. Fortuna, G. Muscato, and M. G. Xibilia, "Attitude feedforward neural controller in quaternion algebra," Intelligent Automation and Soft Computing, vol. 5, no. 3, pp. 191-199, 1999.

[8] G. Huskić, S. Buck, and A. Zell, "GeRoNa: generic robot navigation: a modular framework for robot navigation and control," Journal of Intelligent and Robotic Systems: Theory and Applications, vol. 95, no. 2, pp. 419-442, 2019.

[9] A. Sgorbissa, "Integrated robot planning, path following, and obstacle avoidance in two and three dimensions: wheeled robots, underwater vehicles, and multicopters," The International Journal of Robotics Research, vol. 38, no. 7, pp. 853-876, 2019.

[10] P. Arena, S. Fazzino, L. Fortuna, and P. Maniscalco, "Game theory and non-linear dynamics: the Parrondo paradox case study," Chaos, Solitons and Fractals, vol. 17, no. 2-3, pp. 545-555, 2003.

[11] A. Scheidler, A. Brutschy, E. Ferrante, and M. Dorigo, "The k-unanimity rule for self-organized decision-making in swarms of robots," IEEE Transactions on Cybernetics, vol. 46, no. 5, pp. 1175-1188, 2016.

[12] R. Groß, A. Kolling, S. Berman, A. Martinoli, E. Frazzoli, and F. Matsuno, "Guest editorial: special issue on distributed robotics-from fundamentals to applications," Autonomous Robots, vol. 42, no. 8, pp. 1521-1523, 2018.

[13] V. J. Koeman, H. J. Griffioen, D. C. Plenge, and K. V. Hindriks, "Designing a cognitive agent connector for complex environments: a case study with starcraft," International Workshop on Engineering Multi-Agent Systems, EMAS, Stockholm; Sweden, pp. 302-319, 2019.

[14] R. Limosani, A. Manzi, A. Faggiani et al., "Low-cost solution in international robotic challenge: lessons learned by Tuscany robotics team at ERL emergency robots 2017," Journal of Field Robotics, vol. 36, no. 3, pp. 587-601, 2019.

[15] A. E. Gorodetskiy and I. L. Tarasova, "Situational control a group of robots based on SEMS," Studies in Systems, Decision and Control, Springer, Cham, Switzerland, pp. 9-18, 2019.

[16] D.-S. Jang, H.-J. Chae, and H.-L. Choi, "Optimal controlbased UAV path planning with dynamically-constrained TSP with neighborhoods," in Proceedings of the International Conference on Control, Automation and Systems (ICCAS), pp. 373-378, IEEE, Jeju, Republic of Korea, October 2017.

[17] K. Gong and C. Chen, "Multiple-attribute decision making based on equivalence consistency under probabilistic linguistic dual hesitant fuzzy environment," Engineering Applications of Artificial Intelligence, vol. 85, pp. 393-401, 2019.
[18] M. Akram and F. Zafar, "Bipolar fuzzy soft graphs," Hybrid Soft Computing Models Applied to Graph Theory, Springer, Cham, Switzerland, pp. 353-370, 2020.

[19] N. Masoudi, G. M. Fadel, and M. M. Wiecek, "Planning the shortest path in cluttered environments: a review and a planar convex hull-based approach," Journal of Computing and Information Science in Engineering, vol. 19, no. 4, 2019.

[20] N. Nedjah and L. S. Junior, "Review of methodologies and tasks in swarm robotics towards standardization," Swarm and Evolutionary Computation, vol. 50, 2019.

[21] N. Yao, D. Miao, W. Pedrycz, H. Zhang, and Z. Zhang, "Causality measures and analysis: a rough set framework," Expert Systems with Applications, vol. 136, pp. 187-200, 2019.

[22] U. Bentkowska, "Fuzzy sets and their extensions," Studies in Fuzziness and Soft Computing, vol. 378, pp. 3-23, 2020. 